International Journal of Pure and Applied Mathematics

Volume 86 No. 2 2013, 333-344

ISSN: 1311-8080 (printed version); ISSN: 1314-3395 (on-line version)

url: http://www.ijpam.eu

doi: http://dx.doi.org/10.12732/ijpam.v86i2.8

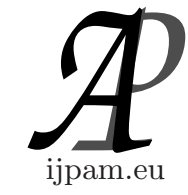

\title{
ON THE REGULAR ELEMENTS OF RINGS IN WHICH THE PRODUCT OF ANY TWO ZERO DIVISORS LIES IN THE GALOIS SUBRING
}

Owino Maurice Oduor ${ }^{1}$, Omamo Aggrey Libendi², Musoga Christopher ${ }^{3}$

${ }^{1}$ Department of Mathematics and Computer Science

University of Kabianga

P.O. Box 2030-20200, Kericho, KENYA

${ }^{2,3}$ Department of Mathematics

Masinde Muliro University of Science and Technology

P.O. Box 190-50100, Kakamega, KENYA

\begin{abstract}
Suppose $R$ is a completely primary finite ring in which the product of any two zero divisors lies in the Galois (coefficient) subring. We construct $R$ and find a generalized characterization of its regular elements.
\end{abstract}

AMS Subject Classification: 13M05, 16P10, 16U60, 13E10, 16N20

Key Words: unit groups, completely primary finite rings

\section{Introduction}

Unless otherwise stated, $J(R)$ shall denote the Jacobson radical of a completely primary finite ring $R$. We shall denote the coefficient (Galois) subring of $R$ by $R^{\prime}$. The set of all the regular elements in $R$ shall be denoted by $V(R)$. The rest of the notations shall be adopted from [1].

An element $x \in R$ is called regular if there exists $y \in R$ such that $x=x^{2} y$. The element $y$ is called a von Neumann inverse of $x$, see e.g [2]. It is well known that in any local ring, a regular element is either a unit or zero. Further details on the classes of completely primary finite rings consideredin this work may be

Received: January 28, 2013

(c) 2013 Academic Publications, Ltd.

$\S$ Correspondence author

url: www.acadpubl.eu 
obtained in [3] and [4].

\section{The Construction}

Let $R^{\prime}$ be the Galois ring of the form $G R\left(p^{n r}, p^{n}\right)$. For each $i=1, \ldots, h$, let $u_{i} \in J(R)$, such that $U$ is an $h$-dimensional $R^{\prime}$-module generated by $\left\{u_{1}, \ldots, u_{h}\right\}$ so that $R=R^{\prime} \oplus U$ is an additive group. On this group, define multiplication by the following relations:

(i) If $n=2$, then $u_{i} u_{j}=p \alpha_{i j}, u_{i}^{3}=u_{i}^{2} u_{j}=u_{i} u_{j}^{2}=0, u_{i} r^{\prime}=\left(r^{\prime}\right)^{\sigma_{i}} u_{i}$

(ii) If $n \geq 3$, then

$p^{n-1} u_{i}=0, u_{i} u_{j}=p^{2} \alpha_{i j}+p^{n-1} \beta_{i j}, u_{i}^{n}=u_{i}^{n-1} u_{j}=u_{i} u_{j}^{n-1}=0, u_{i} r^{\prime}=\left(r^{\prime}\right)^{\sigma_{i}} u_{i}$,

where $r^{\prime}, \alpha_{i j} \in R^{\prime}, \beta_{i j} \in R^{\prime} / p R^{\prime}, 1 \leq i, j \leq h$ and $\sigma_{i}$ is the automorphism associated with $u_{i}$. Further, let $p u_{i}=u_{i} u_{j}=0$, when $u_{i} \in U$.

From the given multiplication in $R$, we notice that $r^{\prime}, s^{\prime} \in R^{\prime}, \gamma_{i}, \lambda_{i} \in F_{0}$ are elements of $R$, then

$$
\begin{aligned}
& \left(r^{\prime}+\sum_{i=1}^{h} \lambda_{i} u_{i}\right)\left(s^{\prime}+\sum_{i=1}^{h} \lambda_{i} u_{i}\right) \\
& =r^{\prime} s^{\prime}+p^{n-1} \sum_{i, j=1}^{h} \xi_{i j}\left(\lambda_{i}\left(\gamma_{j}\right)^{\sigma_{i}}+p R^{\prime}\right)+\sum_{i=1}^{h}\left[\left(r^{\prime}+p R^{\prime}\right) \gamma_{i}+\lambda_{i}\left(s^{\prime}+p R^{\prime}\right)^{\sigma_{i}}\right] u_{i},
\end{aligned}
$$

where $r^{\prime}, s^{\prime} \in R^{\prime}, \lambda_{i}, \gamma_{i} \in F_{0}, \xi_{i j} \in R^{\prime} / p R^{\prime}$. It is easy to verify that the given multiplication turns $R$ into a ring with identity $(1,0, \ldots, 0)$. We also notice that $p^{n-1} \in(J(R))^{2}$ when $\operatorname{char} R=\operatorname{char} R^{\prime}=p^{n}, n \geq 2$. Specifically, $p \in(J(R))^{2}$ when $n=2$.

\section{Preliminary Results}

Lemma 1. The ring described by the construction is commutative iff $\sigma_{i}=i d_{R^{\prime}}$ for each $i=1, \ldots, h$.

Proof. It is evident

Remark: If $n=2$, then the construction yields rings satisfying the properties

$$
J(R)=p R^{\prime} \oplus U
$$




$$
\begin{aligned}
& (J(R))^{2}=p R^{\prime} \\
& (J(R))^{3}=(0) .
\end{aligned}
$$

On the other hand, if $n \geq 3$, then $J(R)=p R^{\prime} \oplus U$

$$
\begin{gathered}
(J(R))^{n-1}=p^{n-1} R^{\prime} \\
(J(R))^{n}=(0) .
\end{gathered}
$$

Now, consider a commutative ring $R$ from the class of rings described by the construction, we notice that

$$
\begin{gathered}
R=R^{\prime} \oplus \sum_{i=1}^{h} R^{\prime} u_{i} \\
J(R)=p R^{\prime} \oplus \sum_{i=1}^{h} R^{\prime} u_{i} .
\end{gathered}
$$

So

$$
1+J(R)=1+p R^{\prime} \oplus \sum_{i=1}^{h} R^{\prime} u_{i} .
$$

Further, $V(R)=R^{*} \cup\{0\}=\left(R^{*} / 1+J(R)\right) .(1+J(R)) \cup\{0\}=<a>.(1+$ $J(R)) \cup\{0\} \cong<a>\times(1+J(R)) \cup\{0\} \cong \mathbf{Z}_{p^{r}-1} \times(1+J(R)) \cup\{0\}$. It therefore suffices to determine the structure of $1+J(R)$.

Proposition 1. For each prime integer $p, 1+p R^{\prime}$ is a subgroup of $1+J(R)$.

Proposition 2. For each prime integer $p, 1+p R^{\prime} \oplus R^{\prime} u_{1}$ is a subgroup of $1+J(R)$.

Proposition 3. For each $1 \leq j \leq h, 1+\sum_{j=1}^{h} \oplus R^{\prime} u_{j}$ is a subgroup of $1+J(R)$. result

Since the two sided annihilator $\operatorname{ann}(J(R))=p^{n-1} R^{\prime}$, we state the following

Proposition 4. $1+\operatorname{ann}(J(R)) \leq 1+p R^{\prime} \leq 1+J(R)$.

Proof. It suffices to prove that $1+\operatorname{ann}(J(R)) \leq 1+p R^{\prime}$. Clearly $1+$ $\operatorname{ann}(J(R))=1+p^{n-1} R^{\prime}, \forall n \geq 2$. Now, for $r^{\prime}, s^{\prime} \in R^{\prime}$, let $1+p^{n-1} r^{\prime}, 1+p^{n-1} s^{\prime} \in$ $1+\operatorname{ann}(J(R))$. Then

$$
\left(1+p^{n-1} r^{\prime}\right)\left(1+p^{n-1} s^{\prime}\right)^{-1}
$$




$$
\begin{aligned}
& =\left(1+p^{n-1} r^{\prime}\right)\left(1-p^{n-1} s^{\prime}\right) \\
& =1+p^{n-1}\left(r^{\prime}-s^{\prime}\right) \in 1+\operatorname{ann}(J(R))
\end{aligned}
$$

Proposition 5. Let $p=2$. Then the 2- group $1+J(R)$ is a direct product of the subgroups $1+p R^{\prime} \oplus R^{\prime} u_{1}$ by $1+\sum_{i=1}^{h} \oplus R^{\prime} u_{i}$, with $h \geq 2$.

Proposition 6. Let $p \neq 2$. The $p-\operatorname{group} 1+J(R)$ is a direct product of the subgroups $1+p R^{\prime}$ by $1+\sum_{i=1}^{h} \oplus R^{\prime} u_{i}$.

Proposition 7. Let $U$ be a finitely generated $R^{\prime}-$ module. If $U$ is generated by $\left\{u_{1}, \ldots, u_{h}\right\}$, then $\left\{u_{1}, u_{1}+u_{2}, \ldots, u_{h-1}+u_{h}\right\}$ also generates $U$.

Proof. If $U$ is a finitely generated $R^{\prime}-$ module, then there exist $\alpha_{1}, \ldots, \alpha_{h} \in$ $R^{\prime}$, such that every $u \in U$ can be expressed in the form $u=\sum_{i=1}^{h} \alpha_{i} u_{i}$. But $\sum_{i=1}^{h} \alpha_{i} u_{i}=\left(\alpha_{1}-\alpha_{2}+\ldots+(-1)^{h+1} \alpha_{h}\right) u_{1}+\left(\alpha_{2}-\alpha_{3}+\ldots+(-1)^{h} \alpha_{h}\right)\left(u_{1}+\right.$ $\left.u_{2}\right)+\ldots+\left(\alpha_{h-1}-\alpha_{h}\right)\left(u_{h-2}+u_{h-1}\right)+\alpha_{h}\left(u_{h-1}+u_{h}\right)$. Since all the coefficients $\alpha_{1}-\alpha_{2}+\ldots+(-1)^{h+1} \alpha_{h}, \alpha_{2}-\alpha_{3}+\ldots+(-1)^{h} \alpha_{h}, \ldots, \alpha_{h-1}-\alpha_{h}$ and $\alpha_{h}$ belong to $R^{\prime}$, it follows that $\left\{u_{1}, u_{1}+u_{2}, \ldots, u_{h-1}+u_{h}\right\}$ generates $U$.

Proposition 8. Let $R$ be a commutative finite ring from the class of finite rings described by the construction. If $U$ is generated by $\left\{u_{1}, \ldots, u_{h}\right\}$, then it is also generated by $\left\{u_{1}, u_{1}+u_{2}, \ldots, u_{1}+u_{2}+\ldots+u_{h}\right\}$.

\section{Main Results}

Proposition 9. Let $R$ be a commutative finite ring from the class of finite rings described by the construction. If $h \geq 1$ and $\operatorname{char} R=p^{2}$, then

$$
1+J(R) \cong\left\{\begin{array}{l}
\boldsymbol{Z}_{4}^{r} \times\left(\boldsymbol{Z}_{2}^{r}\right)^{h-1} \text { if } p=2 \\
\boldsymbol{Z}_{p}^{r} \times\left(\boldsymbol{Z}_{p}^{r}\right)^{h} \text { if } p \neq 2
\end{array}\right.
$$

Proof. Let $\lambda_{1}, \ldots, \lambda_{r} \in R^{\prime}$ with $\lambda_{1}=1$ such that $\overline{\lambda_{1}}, \ldots, \overline{\lambda_{r}} \in R^{\prime} / p R^{\prime}$ form a basis for $R^{\prime} / p R^{\prime}$ regarded as a vector space over its prime subfield $G F(p)$. Since the two cases do not overlap, we treat them in turn.

Case (i): $p=2$.

We notice that, for every $\nu=1, \ldots, r$ and $u_{1} \in J(R)-(J(R))^{2}$,

$$
\begin{aligned}
& \left(1+\lambda_{\nu} u_{1}\right)^{2}=1+2 \lambda_{\nu}^{2}+2 \lambda_{\nu} u_{1} \\
& \quad=1+2 \lambda_{\nu}^{2}, \text { since } 2 \in(J(R))^{2} \text { and } 2 u_{1}=0 .
\end{aligned}
$$


Now,

$$
\begin{aligned}
& \left(1+2 \lambda_{\nu}^{2}\right)\left(1+\lambda_{\nu} u_{1}\right)=1+2 \lambda_{\nu}^{2}+\left(\lambda_{\nu}+2 \lambda_{\nu}^{3}\right) u_{1} \\
& \quad=1+2 \lambda_{\nu}^{2}+\lambda_{\nu} u_{1}, \text { since } 2 \in(J(R))^{2} \text { and } 2 u_{1}=0 .
\end{aligned}
$$

But then,

$$
\begin{aligned}
(1+ & \left.2 \lambda_{\nu}^{2}+\lambda_{\nu} u_{1}\right)\left(1+\lambda_{\nu} u_{1}\right) \\
& =1+2^{2} \lambda_{\nu}^{2}+2\left(\lambda_{\nu}+\lambda_{\nu}^{3}\right) u_{1} \\
& =1, \text { since } 2 \in(J(R))^{2} \text { and } 2 u_{1}=0 .
\end{aligned}
$$

Also, for each $u_{i} \in J(R)-(J(R))^{2}, 1 \leq i \leq h-1,\left(1+\lambda_{\nu} u_{i}+\lambda_{\nu} u_{i+1}\right)^{2}=1+$ $2\left(2^{2} \lambda_{\nu}^{2}\right)+2 \lambda_{\nu}\left(u_{i}+u_{i+1}\right)=1$, since $(J(R))^{3}=(0)$ so that $2^{3}=0,2 u_{i}=2 u_{i+1}=0$, as $2 \in(J(R))^{2}$.

So, for each $\nu=1, \ldots, r$ and $1 \leq i \leq h-1,\left(1+\lambda_{\nu} u_{1}\right)^{4}=1,\left(1+\sum_{i=1}^{h-1} \lambda_{\nu}\left(u_{i}+\right.\right.$ $\left.\left.u_{i+1}\right)\right)^{2}=1$.

For positive integers $\alpha_{\nu}, \beta_{i \nu}$ with $\alpha_{\nu} \leq 4, \beta_{i \nu} \leq 2(1 \leq i \leq h-1,1 \leq \nu \leq$ $r)$, we notice that the equation

$$
\prod_{\nu=1}^{r}\left\{\left(1+\lambda_{\nu} u_{1}\right)^{\alpha_{\nu}}\right\} \cdot \prod_{i=1}^{h-1} \prod_{\nu=1}^{r}\left\{\left(1+\lambda_{\nu}\left(u_{i}+u_{i+1}\right)\right)^{\beta_{i \nu}}=\{1\}\right.
$$

will imply $\alpha_{\nu}=4$ and $\beta_{i \nu}=2,1 \leq i \leq h-1$. If we set

$$
\begin{gathered}
T_{\nu}=\left\{\left(1+\lambda_{\nu} u_{1}\right)^{\alpha} \mid \alpha=1, \ldots, 4\right\}, \\
S_{i \nu}=\left\{\left(1+\lambda_{\nu}\left(u_{i}+u_{i+1}\right)^{\beta_{i}} \mid \beta_{i}=1,2\right\}\right.
\end{gathered}
$$

we see that $T_{\nu}, S_{i \nu}$ are all cyclic subgroups of the group $1+J(R)$ and they are of the orders indicated in their definition. Since

$$
\prod_{\nu=1}^{r}\left|<1+\lambda_{\nu} u_{1}>\right| \cdot \prod_{i=1}^{h-1} \prod_{\nu=1}^{r}\left|<1+\lambda_{\nu}\left(u_{i}+u_{i+1}\right)>\right|=2^{(h+1) r}
$$

and the intersection of any pair of the cyclic subgroups gives the identity group, the product of the $h r$ subgroups $T_{\nu}, S_{i \nu}, 1 \leq i \leq h-1$ is direct. Therefore, their product exhausts the group $1+J(R)$.

Case (ii): $p$ is odd.

If $\nu=1, \ldots, r$ and $u_{i} \in J(R)-(J(R))^{2}, 1 \leq i \leq h-1$,

$$
\left(1+p \lambda_{\nu}\right)^{p}=1+p^{2} \lambda_{\nu}+\frac{p(p-1)}{2}\left(p \lambda_{\nu}\right)^{2}+\ldots+\left(p \lambda_{\nu}\right)^{p}
$$




$$
=1, \text { since } \operatorname{char} R=p^{2} \text {. }
$$

Also,

$$
\left(1+\lambda_{\nu} u_{1}\right)^{p}=\left(1+\sum_{i=1}^{2} \lambda_{\nu} u_{i}\right)^{p}=\ldots=\left(1+\sum_{i=1}^{h} \lambda_{\nu} u_{i}\right)^{p}=1 .
$$

For positive integers $\alpha_{\nu}, \beta_{i \nu}$ with $\alpha_{\nu} \leq p, \beta_{i \nu} \leq p(1 \leq i \leq h, 1 \leq \nu \leq r)$, we notice that the equation

$$
\prod_{\nu=1}^{r}\left\{\left(1+p \lambda_{\nu}\right)^{\alpha_{\nu}}\right\} \cdot \prod_{i=1}^{h} \prod_{\nu=1}^{r}\left\{\left(1+\sum_{j=1}^{i} \lambda_{\nu} u_{j}\right)^{\beta_{i \nu}}\right\}=\{1\}
$$

will imply $\alpha_{\nu}=\beta_{i \nu}=p, 1 \leq i \leq h$. If we set

$$
\begin{gathered}
T_{\nu}=\left\{\left(1+p \lambda_{\nu}\right)^{\alpha} \mid \alpha=1, \ldots, p\right\}, \\
S_{i \nu}=\left\{\left(1+\sum_{j=1}^{i} \lambda_{\nu} u_{j}\right)^{\beta_{i}} \mid \beta_{i}=1, \ldots, p\right\}
\end{gathered}
$$

we see that $T_{\nu}, S_{i \nu}$ are all cyclic subgroups of the group $1+J(R)$ and they are of the orders indicated in their definition. Since

$$
\prod_{\nu=1}^{r}\left|<1+p \lambda_{\nu}>\right| \cdot \prod_{i=1}^{h} \prod_{\nu=1}^{r}\left|<1+\sum_{j=1}^{i} \lambda_{\nu} u_{j}>\right|=p^{(h+1) r}
$$

and the intersection of any pair of the cyclic subgroups gives the identity group, the product of the $(h+1) r$ subgroups $T_{\nu}, S_{i \nu}, 1 \leq i \leq h$ is direct. Therefore, their product exhausts the group $1+J(R)$.

Proposition 10. Let $R$ be a commutative finite ring from the class of finite rings given by the construction. If $h \geq 1, r>1$ and char $R=p^{3}$, then

$$
1+J(R) \cong\left\{\begin{array}{l}
\boldsymbol{Z}_{2}^{r} \times \boldsymbol{Z}_{4}^{r} \times\left(\boldsymbol{Z}_{2}^{r}\right)^{h-1} \text { if } p=2 \\
\boldsymbol{Z}_{p^{2}}^{r} \times\left(\boldsymbol{Z}_{p}^{r}\right)^{h} \text { if } p \neq 2
\end{array}\right.
$$

Proof. Let $\lambda_{1}, \ldots, \lambda_{r} \in R^{\prime}$ with $\lambda_{1}=1$ such that $\overline{\lambda_{1}}, \ldots, \overline{\lambda_{r}} \in R^{\prime} / p R^{\prime}$ form a basis for $R^{\prime} / p R^{\prime}$ regarded as a vector space over its prime subfield $G F(p)$. We treat the two cases in turn.

Case (i): $p=2$.

We notice that for every $\nu=1, \ldots, r$ and $u_{1} \in J(R)-(J(R))^{2}$,

$$
\left(-1+4 \lambda_{\nu}\right)^{2}=1-2^{3} \lambda_{\nu}+2^{4} \lambda_{\nu}^{2}
$$




$$
=1 \text {, since } \operatorname{char} R=2^{3} \text {. }
$$

Also

$$
\begin{gathered}
\left(1+\lambda_{\nu} u_{1}\right)^{2}=1+2^{2} \lambda_{\nu}^{2}+2 \lambda_{\nu} u_{1} \\
=1+2^{2} \lambda_{\nu}^{2}, \text { since } 2 u_{1}=0 .
\end{gathered}
$$

But then,

$$
\begin{gathered}
\left(1+2^{2} \lambda_{\nu}^{2}\right)^{2}=1+2^{3} \lambda_{\nu}^{2}+2^{4} \lambda_{\nu}^{4} \\
=1, \text { since } \operatorname{char} R=2^{3} .
\end{gathered}
$$

It is also easy to see that, for each $\nu=1, \ldots, r, 1 \leq i \leq h-1,\left(1+\lambda_{\nu}\left(u_{i}+u_{i+1}\right)\right)^{2}=$ 1.

For positive integers $\alpha_{\nu}, \beta_{\nu}, \kappa_{i \nu}$ with $\alpha_{\nu} \leq 2, \beta_{\nu} \leq 4, \kappa_{i \nu} \leq 2,(1 \leq i \leq$ $h-1,1 \leq \nu \leq r)$, we notice that the equation

$$
\prod_{\nu=1}^{r}\left\{\left(-1+4 \lambda_{\nu}\right)^{\alpha_{\nu}}\right\} \cdot \prod_{\nu=1}^{r}\left\{\left(1+\lambda_{\nu} u_{1}\right)^{\beta_{\nu}}\right\} \cdot \prod_{i=1}^{h-1} \prod_{\nu=1}^{r}\left\{\left(1+\lambda_{\nu}\left(u_{i}+u_{i+1}\right)\right)^{\kappa_{i \nu}}=\{1\}\right.
$$

will imply $\alpha_{\nu}=2$ and $\beta_{\nu}=4, \kappa_{i \nu}=2,1 \leq i \leq h-1$. If we set

$$
\begin{gathered}
H_{\nu}=\left\{\left(-1+4 \lambda_{\nu}\right)^{\alpha} \mid \alpha=1,2\right\}, \\
T_{\nu}=\left\{\left(1+\lambda_{\nu} u_{1}\right)^{\beta} \mid \beta=1, \ldots, 4\right\}, \\
S_{i \nu}=\left\{\left(1+\lambda_{\nu}\left(u_{i}+u_{i+1}\right)^{\kappa_{i}} \mid \kappa_{i}=1,2\right\}\right.
\end{gathered}
$$

we see that $H_{\nu}, T_{\nu}, S_{i \nu}$ are all cyclic subgroups of the group $1+J(R)$ and they are of the orders indicated in their definition. Since

$$
\prod_{\nu=1}^{r}\left|<-1+4 \lambda_{\nu}>\right| \cdot \prod_{\nu=1}^{r}\left|<1+\lambda_{\nu} u_{1}>\right| \cdot \prod_{i=1}^{h-1} \prod_{\nu=1}^{r}\left|<1+\lambda_{\nu}\left(u_{i}+u_{i+1}\right)>\right|=2^{(h+2) r}
$$

and the intersection of any pair of the cyclic subgroups gives the identity group, the product of the $(h+1) r$ subgroups $H_{\nu}, T_{\nu}, S_{i \nu}, 1 \leq i \leq h-1$ is direct. Therefore, their product exhausts the group $1+J(R)$.

Case (ii): $p$ is odd.

Here, we notice that

$$
\left(1+p \lambda_{\nu}\right)^{p^{2}}=1,\left(1+\lambda_{\nu} u_{1}\right)^{p}=\left(1+\sum_{i=1}^{2} \lambda_{\nu} u_{i}\right)^{p}=\ldots=\left(1+\sum_{i=1}^{h} \lambda_{\nu} u_{i}\right)^{p}=1 .
$$


Now, for positive integers $\alpha_{\nu}, \beta_{i \nu}$ with $\alpha_{\nu} \leq p^{2}, \beta_{i \nu} \leq p,(1 \leq i \leq h, 1 \leq \nu \leq$ $r$ ), we notice that the equation

$$
\prod_{\nu=1}^{r}\left\{\left(1+p \lambda_{\nu}\right)^{\alpha_{\nu}}\right\} \cdot \prod_{i=1}^{h} \prod_{\nu=1}^{r}\left\{\left(1+\sum_{j=1}^{i} \lambda_{\nu} u_{j}\right)^{\beta_{i \nu}}\right\}=\{1\}
$$

will imply $\alpha_{\nu}=p^{2}, \beta_{i \nu}=p$ for $1 \leq \nu \leq r$ and $1 \leq i \leq h$. The rest of the proof is similar to Case (ii) in the previous Proposition.

Proposition 11. Let $R$ be a commutative finite ring from the class of finite rings described by the construction. If $h \geq 1, r=1$ and char $R=p^{n}$, $n \geq 4$, then

$$
1+J(R) \cong\left\{\begin{array}{l}
\boldsymbol{Z}_{2} \times \boldsymbol{Z}_{2} \times \boldsymbol{Z}_{2^{n-2}} \times\left(\boldsymbol{Z}_{2}\right)^{h-1} \text { if } p=2 \\
\boldsymbol{Z}_{p^{n-1}} \times\left(\boldsymbol{Z}_{p}\right)^{h} \text { if } p \neq 2
\end{array}\right.
$$

Proof. Case (i): $p=2$.

Consider the element $1+2 t+u_{1}$, where $t=n-4, n \geq 4$, then $o\left(1+2 t+u_{1}\right)=$ $2^{n-2}$. The elements $-1+2^{n-1}$ and $-1+2^{n-2}+u_{1}$ are each of order 2 . Also, the elements $1+u_{1}+u_{2}, 1+u_{2}+u_{3}, \ldots, 1+u_{h-1}+u_{h}$ are each of order 2 . Now, the mentioned elements generate cyclic subgroups of $1+J(R)$. Since $\left|<1+2 t+u_{1}>\right|$ $\cdot\left|<-1+2^{n-1}>\right| .\left|<-1+2^{n-2}+u_{1}>\right| \cdot \prod_{j=2}^{h}\left|<1+u_{j-1}+u_{j}>\right|=2^{n+h-1}$, and the intersection of any pair of the cyclic subgroups gives the identity group, $<1+2 t+u_{1}>\times<-1+2^{n-1}>\times<-1+2^{n-2}+u_{1}>\times<1+u_{1}+u_{2}>$ $\times \ldots \times<1+u_{h-1}+u_{h}>$ is a direct product.

Case (ii): $p \neq 2$.

Here, the element $1+p$ is of order $p^{n-1}$ while the elements $1+u_{1}, 1+$ $\sum_{i=1}^{2} u_{i}, \ldots, 1+\sum_{i=1}^{h} u_{i}$ are each of order $p$. The given elements generate cyclic subgroups of the group $1+J(R)$. Since

$$
|<1+p>| \cdot \prod_{\iota=1}^{h}\left|<1+\sum_{i=1}^{\iota} u_{i}>\right|=p^{n+h-1},
$$

and the intersection of any pair of the cyclic subgroups gives the identity group, $<1+p>\times<1+u_{1}>\times<1+\sum_{i=1}^{2} u_{i}>\times \ldots \times<1+\sum_{i=1}^{h} u_{i}>$ is a direct product.

Proposition 12. Let $R$ be a commutative finite ring from the class of finite rings described by the construction. If $h \geq 1, r>1$ and $\operatorname{char} R=p^{4}$, then

$$
1+J(R) \cong\left\{\begin{array}{l}
\boldsymbol{Z}_{2} \times \boldsymbol{Z}_{2} \times \boldsymbol{Z}_{4} \times \boldsymbol{Z}_{2}^{r-1} \times \boldsymbol{Z}_{8}^{r-1} \times\left(\boldsymbol{Z}_{2}^{r}\right)^{h-1} \text { if } p=2 \\
\boldsymbol{Z}_{p^{3}} \times\left(\boldsymbol{Z}_{p}^{r}\right)^{h} \text { if } p \neq 2
\end{array}\right.
$$


Let $\lambda_{1}, \ldots, \lambda_{r} \in R^{\prime}$ with $\lambda_{1}=1$ such that $\overline{\lambda_{1}}, \ldots, \overline{\lambda_{r}} \in R^{\prime} / p R^{\prime}$ form a basis for $R^{\prime} / p R^{\prime}$ regarded as a vector space over its prime subfield $G F(p)$. We treat the two cases in turn.

Case (i): $p=2$.

Clearly,

$$
\begin{gathered}
\left(-1+2^{3} \lambda_{1}\right)^{2}=1,\left(-1+2^{2} \lambda_{1}+\lambda_{1} u_{1}\right)^{2}=1,\left(-1+2^{3}\left(\lambda_{1}+\lambda_{2}\right)+\lambda_{2} u_{1}\right)^{4}=1 \\
\left(1+2^{2}\left(\lambda_{1}+\lambda_{2}\right)+\lambda_{2} u_{1}\right)^{2}=\left(1+2^{2}\left(\lambda_{1}+\lambda_{3}\right)+\left(\lambda_{2}+\lambda_{3}\right) u_{1}\right)^{2}=\ldots= \\
\left(1+2^{2}\left(\lambda_{1}+\lambda_{r}\right)+\left(\lambda_{2}+\ldots+\lambda_{r}\right) u_{1}\right)^{2}=1,\left(1+2 \lambda_{\nu}+\lambda_{\nu} u_{1}\right)^{8}=1, \\
\left(1+\lambda_{\nu} u_{j-1}+\lambda_{\nu} u_{j}\right)^{2}=1, \quad 2 \leq j \leq h .
\end{gathered}
$$

For positive integers $\alpha, \beta, \kappa, \gamma_{s}, \tau_{\nu}, \omega_{i \nu}$ with $\alpha \leq 2, \beta \leq 2, \kappa \leq 4, \gamma_{s} \leq 2$, $\tau_{\nu} \leq 8, \omega_{i \nu} \leq 2,2 \leq s \leq r, 1 \leq \nu \leq r, 1 \leq i \leq h-1$, we notice that the equation $\left\{\left(-1+2^{3} \lambda_{1}\right)^{\alpha}\right\} \cdot\left\{\left(-1+2^{2} \lambda_{1}+\lambda_{1} u_{1}\right)^{\beta}\right\} \cdot\left\{\left(-1+2^{3}\left(\lambda_{1}+\lambda_{2}\right)+\lambda_{2} u_{1}\right)^{\kappa}\right\} \cdot \prod_{\nu=2}^{r}\{(1+$ $\left.\left.2^{2}\left(\lambda_{1}+\lambda_{\nu}\right)+\sum_{\iota=2}^{\nu} \lambda_{\iota} u_{1}\right)^{\gamma_{\nu}}\right\} \cdot \prod\left\{\left(1+2 \lambda_{\nu}+\lambda_{\nu} u_{1}\right)^{\tau_{\nu}}\right\} \cdot \prod_{i=1}^{h-1} \prod_{\nu=1}^{r}\left\{\left(1+\lambda_{\nu}\left(u_{i}+\right.\right.\right.$ $\left.\left.\left.u_{i+1}\right)\right)^{\omega_{i \nu}}\right\}=\{1\}$, will imply $\alpha=\beta=2, \kappa=4, \gamma_{\nu}=2, \tau_{\nu}=8, \omega_{i \nu}=2$ for every $\nu=1, \ldots, r, \nu=2, \ldots, r$ and $i=1, \ldots, h-1$. If we set

$$
\begin{gathered}
E=\left\{\left(-1+2^{3} \lambda_{1}\right)^{\alpha} \mid \alpha=1,2\right\}, \\
F=\left\{\left(-1+2^{2} \lambda_{1}+\lambda_{1} u_{1}\right)^{\beta} \mid \beta=1,2\right\}, \\
G=\left\{\left(-1+2^{3}\left(\lambda_{1}+\lambda_{2}\right)+\lambda_{2} u_{1}\right)^{\kappa} \mid \kappa=1, \ldots, 4\right\}, \\
\left.H_{\nu}=\left(1+2^{2}\left(\lambda_{1}+\lambda_{\nu}\right)+\sum_{\iota=2}^{\nu} \lambda_{\iota} u_{1}\right)^{\gamma_{\nu}} \mid \gamma_{\nu}=1,2\right\}, \\
K_{\nu}=\left\{\left(1+2 \lambda_{\nu}+\lambda_{\nu} u_{1}\right)^{\tau_{\nu}} \mid 1 \leq \tau_{\nu} \leq 8\right\}, \\
L_{i \nu}=\left\{\left(1+\lambda_{\nu}\left(u_{i}+u_{i+1}\right)\right)^{\omega_{i}}\right\}
\end{gathered}
$$

we see that $E, F, G, H_{2}, \ldots, H_{r}, K_{2}, \ldots, K_{r}, L_{1 \nu}, \ldots, L_{(h-1) \nu}$ are all cyclic subgroups of the group $1+J(R)$ and they are of the orders indicated in their definition.

Since

$$
\begin{gathered}
\left|<-1+8 \lambda_{1}>\right| \cdot\left|<-1+4 \lambda_{1}+\lambda_{1} u_{1}>\right| \cdot\left|<-1+8\left(\lambda_{1}+\lambda_{2}\right)+\lambda_{2} u_{1}>\right| . \\
\prod_{\nu=2}^{r}\left|<1+4\left(\lambda_{1}+\lambda_{\nu}\right)+\sum_{\iota=2}^{\nu} \lambda_{\iota} u_{1}>\right| \cdot \prod_{\nu=2}^{r}\left|<1+2 \lambda_{\nu}+\lambda_{\nu} u_{1}>\right| . \\
\prod_{i=1}^{h-1} \prod_{\nu=1}^{r}\left|<1+\lambda_{\nu}\left(u_{i}+u_{i+1}\right)>\right|=2^{(h+1) r},
\end{gathered}
$$


and the intersection of any pair of the cyclic subgroups gives the identity group, the product of the $1+(h+1) r$ subgroups $E, F, G, H_{2}, \ldots, H_{r}, K_{2}, \ldots, K_{r}, L_{1 \nu}, \ldots$, $L_{(h-1) \nu}$ is direct. Therefore, their product exhausts $1+J(R)$.

Case (ii): $p \neq 2$.

Here the proof is similar to that of Case (ii) in the previous Proposition, with some slight modification.

Proposition 13. Let $R$ be a commutative finite ring from the class of finite rings described by the construction. If $h \geq 1, r>1$ and $\operatorname{char} R=p^{n}$, $n \geq 5$, then

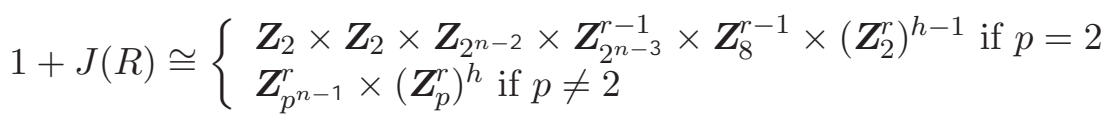

Proof. Let $\lambda_{1}, \ldots, \lambda_{r} \in R^{\prime}$ with $\lambda_{1}=1$ such that $\overline{\lambda_{1}}, \ldots, \overline{\lambda_{r}} \in R^{\prime} / p R^{\prime}$ form a basis for $R^{\prime} / p R^{\prime}$ regarded as a vector space over its prime subfield $G F(p)$. We treat the two cases in turn.

Case (i): $p=2$.

Clearly,

$$
\left(-1+2^{n-1} \lambda_{1}\right)^{2}=1,\left(-1+2^{n-1} \lambda_{1}+2^{n-1} \lambda_{2}\right)^{2}=1,\left(1+2 \lambda_{1}+\lambda_{1} u_{1}\right)^{2^{n-2}}=1
$$

$\left(1+\sum_{\iota=2}^{\nu} \lambda_{\iota} u_{1}\right)^{2^{n-3}}=1,\left(1+4 \lambda_{\nu}+\lambda_{\nu} u_{1}\right)^{2^{n-2}}=1, \nu=2, \ldots, r,\left(1+\lambda_{\nu}\left(u_{i}+\right.\right.$ $\left.\left.u_{i+1}\right)\right)^{2}=1,1 \leq i \leq h-1$ For positive integers $\alpha, \beta, \kappa_{\nu}, \gamma_{\nu}, \tau_{\nu}, \omega_{i \nu}$ with $\alpha \leq 2, \beta \leq 2^{n-2}, \kappa_{\nu} \leq 2, \gamma_{\nu} \leq 2^{n-3}, \tau_{\nu} \leq 2^{n-2}, \omega_{i \nu} \leq 2,1 \leq \nu \leq r, 1 \leq$ $\nu \leq r, 1 \leq i \leq h-1$ we notice that the equation $\left\{\left(-1+2^{n-1} \lambda_{1}\right)^{\alpha}\right\} \cdot\{(1+$ $\left.\left.2 \lambda_{1}+\lambda_{1} u_{1}\right)^{\beta}\right\} \cdot\left\{\left(-1+2^{n-1} \sum_{\nu=1}^{2} \lambda_{\nu}\right)^{\kappa}\right\} \cdot \prod_{\nu=2}^{r}\left\{\left(1+\sum_{\iota=2}^{\nu} \lambda_{\iota} u_{1}\right)^{\gamma_{\nu}}\right\} \cdot \prod\left\{\left(1+4 \lambda_{\nu}+\right.\right.$ $\left.\left.\lambda_{\nu} u_{1}\right)^{\tau_{\nu}}\right\} \cdot \prod_{i=1}^{h-1} \prod_{\nu=1}^{r}\left\{\left(1+\lambda_{\nu}\left(u_{i}+u_{i+1}\right)\right)^{\omega_{i \nu}}\right\}=\{1\}$, will imply $\alpha=2, \beta=2^{n-2}$, $\kappa=2, \gamma_{\nu}=2^{n-3}, \tau_{\nu}=2^{n-2}, \omega_{i \nu}=2$ for every $\nu=2, \ldots, r$ and $i=1, \ldots, h-1$. If we set

$$
\begin{gathered}
E=\left\{\left(-1+2^{n-1} \lambda_{1}\right)^{\alpha} \mid \alpha=1,2\right\} \\
F=\left\{\left(1+2 \lambda_{1}+\lambda_{1} u_{1}\right)^{\beta} \mid \beta=1, \ldots, 2^{n-2}\right\} \\
G=\left\{\left(-1+2^{n-1}\left(\lambda_{1}+\lambda_{2}\right)\right)^{\kappa} \mid \kappa=1,2\right\} \\
\left.H_{\nu}=\left(1+\sum_{\iota=2}^{\nu} \lambda_{\iota} u_{1}\right)^{\gamma_{\nu}} \mid \gamma_{\nu}=1, \ldots, 2^{n-3}\right\}, \\
K_{\nu}=\left\{\left(1+4 \lambda_{\nu}+\lambda_{\nu} u_{1}\right)^{\tau_{\nu}} \mid 1, \ldots, 2^{n-2}\right\}, \\
L_{i \nu}=\left\{\left(1+\lambda_{\nu}\left(u_{i}+u_{i+1}\right)\right)^{\omega_{i}} \mid \omega_{i}=1,2\right\}
\end{gathered}
$$


we see that $E, F, G, H_{2}, \ldots, H_{r}, K_{2}, \ldots, K_{r}, L_{1 \nu}, \ldots, L_{(h-1) \nu}$ are all cyclic subgroups of the group $1+J(R)$ and they are of the orders indicated in their definition.

Since

$$
\begin{gathered}
\left|<-1+2^{n-1} \lambda_{1}>\right| \cdot\left|<1+2 \lambda_{1}+\lambda_{1} u_{1}>\right| \cdot \prod_{\nu=2}^{r}\left|<-1+2^{n-1}\left(\lambda_{1}+\lambda_{2}\right)>\right| . \\
\prod_{\nu=2}^{r}\left|<1+\sum_{\iota=2}^{\nu} \lambda_{\iota} u_{1}>\right| \cdot \prod_{\nu=2}^{r}\left|<1+4 \lambda_{\nu}+\lambda_{\nu} u_{1}>\right| . \\
\prod_{i=1}^{h-1} \prod_{\nu=1}^{r}\left|<1+\lambda_{\nu}\left(u_{i}+u_{i+1}\right)>\right|=2^{(h+n-1) r},
\end{gathered}
$$

and the intersection of any pair of the cyclic subgroups gives the identity group, the product of the $1+(h+1) r$ subgroups $E, F, G, H_{2}, \ldots, H_{r}, K_{2}, \ldots, K_{r}$, $L_{1 \nu}, \ldots, L_{(h-1) \nu}$ is direct. Therefore, their product exhausts $1+J(R)$.

Case (ii): $p \neq 2$.

Here the proof is similar to that of Case (ii) in the previous Proposition, with some slight modification.

We now state the main result.

Theorem 1. The regular elements of the rings described by the construction is given as follows:

i) If $\operatorname{char} R=p^{2}$, then

$$
V(R) \cong\left\{\begin{array}{l}
\boldsymbol{Z}_{2^{r}-1} \times \boldsymbol{Z}_{4}^{r} \times\left(\boldsymbol{Z}_{2}^{r}\right)^{h-1} \cup\{0\} \text { if } p=2 \\
\boldsymbol{Z}_{p^{r}-1} \times \boldsymbol{Z}_{p}^{r} \times\left(\boldsymbol{Z}_{p}^{r}\right)^{h} \cup\{0\} \text { if } p \neq 2
\end{array}\right.
$$

ii) If $\operatorname{char} R=p^{3}$, then

$$
V(R) \cong\left\{\begin{array}{l}
\boldsymbol{Z}_{2^{r}-1} \times \boldsymbol{Z}_{2}^{r} \times \boldsymbol{Z}_{4}^{r} \times\left(\boldsymbol{Z}_{2}^{r}\right)^{h-1} \cup\{0\} \text { if } p=2 \\
\boldsymbol{Z}_{p^{r}-1} \times \boldsymbol{Z}_{p^{2}}^{r} \times\left(\boldsymbol{Z}_{p}^{r}\right)^{h} \cup\{0\} \text { if } p \neq 2
\end{array}\right.
$$

iii) If $\operatorname{char} R=p^{4}$, then

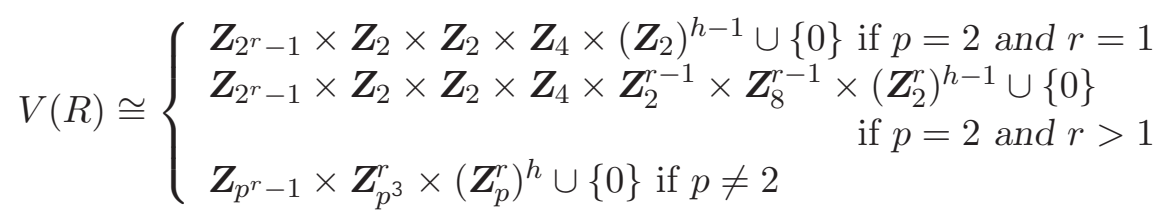


iv) If $\operatorname{char} R=p^{n}, n \geq 5$, then

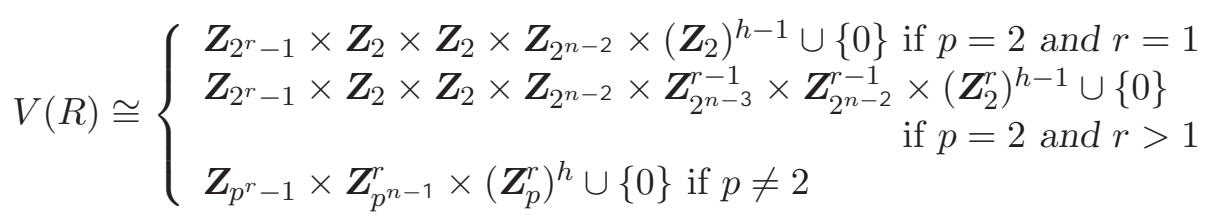

\section{Acknowledgments}

The first author expresses his wholehearted gratitude to DAAD for sponsoring his visit to the African Institute for Mathematical Sciences, South Africa which hosted him during this research.

\section{References}

[1] C.J. Chikunji, Unit groups of cube radical zero commutative completely primary finite rings, International Journal of Mathematics and Mathematical Sciences, 4 (2005), 579-592.

[2] A. Osama, A.O. Emad, On the regular elements in $\mathbf{Z}_{n}$, Turkish Journal of Mathematics, 32 (2008), 31-39.

[3] R. Raghavendran, Finite associative rings, Compositio Math., 21, No. 2 (1969), 195-229.

[4] Y. Alkhamees, Finite completely primary rings in which the product of any two zero divisors of a ring is in its coefficient subring, International Journal of Mathematics and Mathematical Sciences, 17, No. 3 (1994), 463-468. 